\title{
Ngwaga wa Mopedi: seka sa ngwaga wa merafe ye mengwe ya Bibele
}

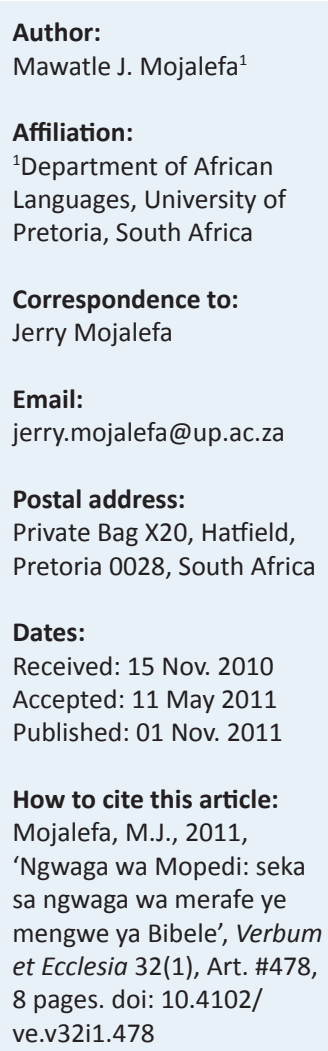

C 2011. The Authors. Licensee: AOSIS OpenJournals. This work is licensed under the Creative Commons Attribution License.

\section{The Pedi year: A comparison with the year of the other biblical nations}

The investigation grounds arguments on facts that there is correlation between Sepedi and Hebrew calendars particularly in relation to similarity as well as difference between the two nations' beliefs which are related to the number (twelve months) of the months of the year. This means that Sepedi and Hebrew believe that the year comprised twelve months. Adding to this, the four Hebrew's early names of the months of the year, namely Abib, Ziv, Ethanim and Bul, of uncertain meaning, differ from the contemporary Hebrew calendar months. This featuristic phenomenon appears also in the Sepedi naming of the months of the year where a month is named after two or more names, such as Dibokwane (February), Hlakola (March), Mopitlo (May), Phupu (June) and Ngwatobošego (June) as well as Mosegamanye (July). The arrangement of the months of the year brings forth certain similarity to the two calendars of these two languages. However, there is difference between the two calendars in relation to the number of the days of the week.

\section{Summary}

Mopedi knows the sun, planets (stars), constellations and stars by names, but has a number of misconceptions different from other biblical nations regarding the universe. Every day is believed to bring a new sun and it is maintained that there are twelve moons (months) which make their regular, appointed appearance. The total number of his or her days a week is six, namely Monday, Tuesday, Wednesday, Thursday, Friday and Saturday.

Furthermore, Mopedi believes that a year comprised twelve months that are related to the moons which make their regular appearance in the unirverse. Observably he or she does not differentiate the planets and the stars because to him or her there is no difference. He or she has given these stars names: Mahlapolana (Mars), Kgogamašego (Vernus), Dithutlwa (Southern Stars), Selemela (Constalation), mošošonono (comet), etc.

On the other hand, according to Kings 4:7 and 1 Chronichles 27:1-15, the Hebrew year followed the W Semitic Calendar with a year of twelve lunar months. However, it is not certain whether the start of the year in spring (Nisan) was for use only, because there is some evidence for the year for civil purposes being sometimes reckoned from the autumn month of Tishri as it is stated in the Chronology of the Old Testament.

The investigation grounds arguments on facts that there is correlation between Sepedi and Hebrew calendars particularly in relation to similarity as well as difference between the two nations' beliefs which are related to the number (twelve months) of the months of the year. This means that Sepedi and Hebrew believe that the year comprised twelve months. Adding to this, the four Hebrew's early names of the months of the year, namely Abib, Ziv, Ethanim and Bul, of uncertain meaning, differ from the contemporary Hebrew calendar months. This featuristic phenomenon appears also in the Sepedi naming of the months of the year where a month is named after two or more names, such as Dibokwane (February), Hlakola (March), Mopitlo (May), Phupu (June) and Ngwatobošego (June) as well as Mosegamanye (July). The arrangement of the months of the year brings forth certain similarity to the two calendars of these two languages. However, there is difference between the two calendars in relation to the number of the days of the week.

\section{Matseno}

Mopedi o tseba letšatši, dipolanete, (dinaledi), moola (sehlopha sa dinaledi) le dingwedi (dikgwedi) ka maina. Le ge go le bjalo o na le kwešišo ye nngwe ye e rilego ya go fapana le ya merafe ye mengwe ya Bibele go ya ka fao, gagolo legohle le hlalošwago le go hlathollwa 
ka gona malebana le kwešišo ya tlholego ya leratadima. O dumela gore letšatši le lengwe le le lengwe le hlaba le letšatši la lona. Ka tsela yeo Mopedi o na le matšatši a a selelago ao a a reetšego maina, e lego Mošupologo, Labobedi, Laboraro, Labone, Labohlano le Mokibelo. Go lemogwa gore matšatši ao a beakantšwe go ya ka palo yeo a latelanago ka yona. Ka go realo beke ya Mopedi e na le matšatši a a selelago, e sego a a šupago go ya ka thulaganyo ya lehono ya matšatši a beke.

Mopedi o holofela gore go na le dingwedi (dikgwedi) tše lesomepedi tšeo di bonalago nako le nako mo leratadimeng mo bophelong bja gagwe. Eupša taba ye bohlokwa ke gore ga a farologantšhe dipolanete le dinaledi ka gobane go yena ka moka ke dinaledi fela. Ebile o fele dinaledi tšeo maina: mahlapolana, kgogamašego, dithutlwa, selemela, bjalobjalo. Go ya ka Mönnig (1988:148) kakanyo ye bjalo e fetotšwe kutšwana ke thuto ye mpsha, le ge e se e fetole kudu tumelo ya gore letšatši, kgwedi le dinaledi di lebane le semoya (seriti), le gore seriti sa dihlolwa tša legodimo se na le khuetšo ye maatla bophelong bja Mopedi, gagologolo ge go hlokometšwe merero ya tlhago ka kakaretšo. Mohlala, (1) ge ngwedi o ka balama o kekemile, o bolela gore kgwedi yeo ke ya komelelo ka gobane go thwe ngwedi o tla o se o ge selo (meetse), (2) ge letšatši/ngwedi le ka swiswala, gona taba yeo e lebane le seka sa lehu la kgoši (ye kgolo) le (3) ge mahlapolane o ka dula ka thokong ya bodikela, o bontšha ngwaga wa pula, mola ge o ka dula ka bohlabela o laetša ngwaga wa kgwakgwa/komelelo, bjalobjalo.

Ka lehlakoreng le lengwe, go ya ka 1 Dikgoši 4:7 le 1 Dikronika 27:1-15, tšhupamabaka ya kgwedi ya Seheberu e theilwe godimo ga ya $W$ Semitic Calendar, moo ngwaga o hlalošwago go ba le dikgwedi tše lesomepedi. Le ge go le bjalo ga go kgonthišo yeo e tiišago gore go thongwa ga ngwaga woo go lebane le nako ya seruthwane (Nisan) ka ge seo e be e le tiragatšo ya tirelo (ritual activity) ya Baheberu, ka lebaka la gore go na le bohlatse bja go thewa godimo ga paka ya go thongwa ga ngwaga, bjoo bo hlalošago maikemišetšo a setšhaba ka go a akanya go lebeletšwe kgwedi ya lehlabula, e lego Tishri; gomme yona taba yeo e lebane le tatelano ya mabaka a Testamente ye Tala.

Go ya ka polelo ya ka godimo go bonagala gabotse gore go na le kwano ye e rilego ya go ba magareng ga tumelo ya Bapedi le Baheberu, ya go lebana le palo ya dikgwedi tše lesomepedi tša ngwaga. Ke go re bobedi merafe ye e dumela gore ngwaga o na le dikgwedi tše lesomepedi. Bjale ge, nyakišišo ye e yo tsinkela kgonthe ya taba yeo go bona ge go le kwano goba phapano magareng ga tirišo ya mabaka a morafe wa Bapedi le merafe yeo ya paka ya Bibele. Ge ditaba tše di nyakišišwa go yo latelwa lenaneo le:

- Dikgwedi tša ngwaga wa Sepedi

- Dikgwedi tša ngwaga wa Seheberu

- Thulaganyo ya dikgwedi

- Dihla tša ngwaga wa Sepedi

- Dihla tša ngwaga wa Seheberu

- Tlhalošo ya maina a dikgwedi tša ngwaga wa Sepedi.

\section{Dikgwedi tša ngwaga wa Sepedi}

Bjalo ka ge nyakišišo ye e lebane le kwano le / goba phapano ya dipaka, go tlo thongwa ka dikgwedi tša Sepedi pele gomme gwa latela tša merafe yeo ye mengwe. Go ya ka mo re šetšego re boletše ka gona ka godimo, go bjalo le go dikgwedi tša ngwaga tša Sepedi ka gobane ke tše lesomepedi ka palo. (Go tlo lemogwa gore dikgwedi tša ngwaga wa Sepedi ga se tša tšo hlalošwa ka botlalo go fihlela nakong ye ya nyakišišo. Rateori yo a šetšego a kgwathile karolwana ye ke Mönnig). Ge a tiiša taba ye Mönnig (1988) o re:

The Pedi know many planets, stars and constellations by name, but have a number of misconceptions regarding the universe. Every day is believed to bring a new sun (letšatši) and it is maintained that there are twelve moons (dikgwedi) which make their regular, appointed appearance.

(Mönnig 1988:148)

Maina a dikgwedi tšeo tša ngwaga a reetšwe go ya ka bohlokwa bja ditiragalo tša ngwaga tše di lebanego le tšona. Go feta fao maina a dikgwedi gammogo le thalošo ya ona, go akaretšwa le mešomo ya paka ya sehla ya go amana le dikgwedi tšeo tše lesomepedi, a magareng ga merero ye bohlokwahlokwa ya thuto ya bafsa ba Bapedi. Kgwedi ye nngwe le ye nngwe e na le leina la go feta le tee, leo le amanywago le ditiragalo tše di itšego tša tlhago, tšeo di diregago pakeng ya kgwedi yeo. Go ya ka Mönnig (1988):

The most important of these names have been chosen for the translation of the names of months with which they have now become associated, and under which headings they may be profitably discussed.

(Mönnig 1988:149)

Se se swanetšego go šetšwa kudu dipolelong tša ka godimo ke gore go tlo lemogwa gore taba ye e ka hlolago kgakanego malebana le maina a a dikgwedi ke ya go lebana le gore kgwedi ye nngwe le ye nngwe e na le leina la go feta le tee.

Maina a dikgwedi tša ngwaga ke ye nngwe ya dikarolo tše bohlokwa mo setšong sa Sepedi. Ge go lebelelwa dikgwedi tša ngwaga ka semmotwana sa Sehlakwana (sehlopha sa Bahlako/Bahlakwana ba semmotwana sa Sepedi), Mopedi wa bogologolo o hlaloša gore ngwaga o thongwa ka kgwedi ya Mopitlo ka gobane ke kgwedi yeo go yona go thongwago karolo ya mathomo ya koma ya banna, e lego ya 'go kgotha' (first session of the initiation) ga babolodi. Ka fao Mopitlo ke kgwedi ye go thwego ke mollwane wa go aroganya dipaka (sessions). Ke go re go thongwa go balwa go tloga ka yona paka yeo go aroganya mengwaga. Mohlomongwe taba ye e tla hlalošega gabotse ge go tsinkelwa tšhupamabaka ya merafe ye mengwe ya Bibele go hlokometšwe dikgwedi tša ngwaga.

\section{Dikgwedi tša ngwaga wa Seheberu}

Ge go balwa Eks. 12:2; 13:3-4; 23:15 le Dt. 16:1,6 go lemogwa gore ge Baheberu ba be ba sa le nageng ya Egipeta ba be ba amanywa le ngwaga wa go ba le dikgwedi tše lesomepedi, moo kgwedi ye nngwe le ye nngwe e bego e na le matšatši 
a masometharo le matšatši a mahlano a tlaleletšo. Ka fao ngwaga o be a na le matšatši a 365 (Herodotus, 2.4). Ka tsela yeo mathomo a dikgwedi tšeo tša ngwaga, goba go ka thwe kgwedi ya mathomo ya ngwaga, e bile ya pakeng ya seruthwane.

Fela ge go lebeledišišwa gape thulaganyo ya dikgwedi tša ngwaga ka Seheberu go tlo lemogwa gore ngwaga wa Seheberu, 'sûaunaÆ', wo o bitšwago bjalo ka lebaka la phetogo goba tatelano ya dihla tša ngwaga, mathomong go be go akanywa gore o thongwa ka kgwedi ya bošupa ya lehlabula ya go bitšwa Tishri Eks. 23:16 le 34:22. Ge Lef. 25:8-10 e tiiša taba yeo e re gape nako yeo e be e lebantšhwa le mathomo a ngwaga wa Sabatha. Ka lehlakoreng le lengwe, Eks. 13:4; 23:15 le kgaolo ya 12 34:18 le Dit. 16:1 di bolela gore leina la kgwedi ya mathomo ya ngwaga wa pele ga bothopša ke Abib. Ka fao ge, go ya ka The New Bible Dictionary (1962:158), go ka hlalošwa tšhupamabaka ya Seheberu ka go re ngwaga wa Seheberu o be o thongwa ka dipaka tše pedi tša go fapana, e lego paka ya seruthwane le ya lehlabula.

Tšhupamabaka ya ngwaga wa Seheberu e theilwe godimo ga dikgwedi tša ngwaga tšeo di bego di thoma ge lebalana le lesesane la ngwedi le be le thoma go bonagala ka bodikela. Letšatši la ngwedi wo mofsa mathomong le be le bonwa bjalo ka seka sa kgethego goba boModimo. Kgwedi (yerah) ye e bolelago ngwedi, e be e akanywa gore e na le matšatši a masomepedisenyane (29) goba a masome a mararo (30) fela. Ke ka fao ngwaga o bego o na le dikgwedi tša ka fase ga tše lesometee (11) ge go bapetšwa le ngwaga wa lehono. Ke ka wona mokgwa woo ngwaga wa Baheberu o bego o na le dikgwedi tše lesometharo ka gona gore letšatši la ngwaga wo mofsa le se tle pele ga seruthwane sa ngwaga, e lego paka ye e lego magareng ga dikgwedi tša Hlakola le Moranang. Le ge go le bjalo ga go mabaka a a hlamatsegilego a saense, ao a tsebegago ao a ka kgonthišago gore go na le mokgwa woo o rilego, woo o bego o dirišwa ke Baheberu go nyalanya tšhupamabaka ya temo le lenaneo la tsela ya lefase le dinaledi ya go dikologa letšatši. Go a kgonega gape gore Baheberu ba ka be ba ile ba diriša se go thwego ke 'Adar ya bobedi' (kgwedi ya lesomepedi) goba 'Elul ya bobedi' (kgwedi ya boselela) mo lenaneong la lefase le dinaledi la go dikologa letšatši la mengwaga ye 3, 6, 11, 14, 17 goba 19. Eupša le ge go le bjalo go na le bohlatse bja gore Baheberu ba dirišitše tsela ya go tlaleletša ngwaga ka dikgwedi tše di itšego morago ga Adar (Nu. 9:11; 2 Ch. 30:2-3; bona gape le 1 Dikg. 12:23-33), le ge e le gore morago ga Nisan (bona 2 Ch. 30:2 le ditemana tša go latela), go dirilwe taba yeo go ekiša ka fao go bego go dirwa ka gona kua Mesopotamia. Go ka kgonthišwa gore tšhupamabaka ya go nepagala e dirišitšwe ka mo pukung ya Jubilees (c. 105 bc) le ya 1 Enoch 72-82.

Go ka gatelelwa gore go lemogwa gore tekananako (equinox) ya lehlabula, e lego se se bitšwago 'go selaganywa ga ngwaga' goba gona 'go hwa ga ngwaga' (bona mo go Eks. 23:16), le tekananako ya seruthwane, se se bitšwago 'go tsošološwa ga ngwaga' (1 Dikg. 20:26; Ch. 36:10) go bile bohlokwa taolong ya tšhupamabaka, gomme bohlokwa bjoo morago bo akaretšwa le mehla ya menyanya ya setšhaba. Ka go realo ngwaga o be o thongwa ka ngwedi wo mofsa wa go ba kgauswi le tekananako ya seruthwane ge letšatši le le ka Aries (Jos., Ant. 3. 201), gammogo le phethagatšo ya Paseka ya letšatši la lesomenne la Nisan gape gammogo le go welana ga go tšwelela ga ngwedi wo o tletšego wa mathomo (bona Eks. 12:2-6).

Godimo ga moo ge go balwa magareng ga Ditestamente go lemogwa gore 'ngwaga wa pušo ya Bagerike' (bona 1 Macc. 1:10) o lebane le paka ya Seleucid yeo, semmušo, e tšwelelago go tloga go letšatši la mathomo la kgwedi ya Macedonia ya go bitšwa Dios ye e lebanego le kgwedi ya Lewedi goba Diphalane mo ngwageng wa 312 Pele ga Kriste. Paka yeo e hlalošwa ka go hlompšha ka botlalo goo go bolelwago mo go 1 Macc., le ge e le gore mo methopong ye mengwe ya go dirišwa mo pukung yeo, e (paka yeo) bolela gore e lebane le kgwedi ya Hlakola goba Moranang go ya ka fao go bego go le ka gona ka ngwaga wa 311 Pele ga Kriste, go fapana le kgwedi yeo ya Lewedi goba Diphalane. Taba yeo e gatelela gore ke khuetšo ya Bababilone yeo e hlotšego gore go akanywe gore Nisane fetoge kgwedi ya mathomo ya ngwaga.

Gape go tlo hlokomelwa go ya pele gore ge go balwa Testamente ye Mpsha gona go tlo itemogelwa gore dinako gantši di fele di akanywa ka go lebanywa le babuši ba Baditšhaba. Mohlala wo mobotse wa go nyalelana le ditaba tše o hwetšwa mo pukung ya Lk. 3:1 le ditemana tša go latela, moo mathomo a thomo ya Johanne Mokolobetše, yeo paka ya yona e hlalošwago, e sego fela 'mo ngwageng wa bolesometlhano wa pušo ya Tiberiase Kesare' (ge go oketšwa ka ditemana tša 27 le 28), a nepišwago le kakanyo ya go humanwa mo go pušo ya peleng ya Seleucid, moo ngwaga wa pušo ye mpsha o nyantšwego go tloga ka kgwedi ya Lewedi goba Diphalane. Ditaba tšeo di kgonthišwa gape ke go bolelwa ka babuši ge bjale ba le maemong a pušo, e ka ba ya sehetene goba ya sedumedi mo nageng ya Jutea le ditikologong tša kgauswi le yona. Godimo ga moo go sa le le dipaka tše dingwe tša go hlagišwa ke babuši ba go swana le Agostose (Lk. 2:1) le Klaudiase (Ditiro 11:28), babušiši ba dilete bjalo ka Khwiriniase (Lk. 2:2) le Galio (Ditiro 18:12), le Herote kgoši ya Bajuta (Mt. 2:1; Lk. 1:5).

Mo dikarolong tše kgolo tša Testamente ye Mpsha, bangwadi ba gona ba ngwadile kudu ka tsela ya go akanya nako go ya ka tšhupamabaka (goba ditšhupamabaka) ya bjale ya Bajuta. Dipaka tšeo di hlalošwa go ya ka menyanya le meletlo ye mengwe ya semoya ya Bajuta, gagologolo ge go balwa Ebangedi ya Bone (bona Jn. 2:13, 23 (Paseka); 5:1 (mohlomongwe gape le Ngwaga wo Mofsa); 6:4 (Paseka); 7:2 (Dikgwerano; mo go temana ya 37 'letšatši la mafelelo, letšatši le legolo la monyanya' ke letšatši la boseswai; bona Lef. 23:36; Nu. 29:35; Ne. 8:18); 10:22; (Kabelo/Boikgafo, ka la 25 Kislew; bona gape 1 Macc. 4:59); 11:55 le ditemana tša go latela. (Paseka), bona gape Mt. 26:2; Mk. 14:1; Lk. 22:1 (Paseka le Senkgwa sa go hloka komelo); Ditiro 2:1 (Tšhologo ya Moyamokgethwa); 12:3 le temana ya go latela. (Paseka le 
Senkgwa sa go hloka komelo); 18:21, (mohlomongwe gape le Paseka); 20:6 (Senkgwa sa go hloka komelo); 20:16 (Tšhologo ya Moyamokgethwa); 27:9 (moo go itima dijo e lego Letšatši la Kgwerano, paka yeo e amanywago le go sesa ka lewatleng la Medithereniene, yeo e amanago le go fela ga marega); 1 Dikron. 16:8 (Tšhologo ya Moyamokgethwa).

Magareng ga matšatši a beke, letšatši leo go bolelwago kutšwana ka ga lona ke Sabatha. Sabatha sa mathomo sa bobedi (Lk. 6:1), go bonagala e le kgopolo yeo tlhalošo ya yona e sa hlwego e kgonagala go hlathollwa ka kgotsofalo. Labohlano ke 'letšatši la Tokišo', ka Segerike e lego gore paraskeue $\mu$, ke go re letšatši pele ga Sabatha, seo ka Segerike se bolelago gore prosabbaton (Mk. 15:42; bona gape le Jn. 19:31). 'Letšatši la Tokišo ya Paseka' (Jn. 19:14) le hlaloša 'Labohlano la beke ya Paseka', seo ka Segerike go thwego ke paraskeueu tou pascha. 'Letšatši la mathomo la beke' leo le bitšwago mia sabbatou or mia toun sabbatounka Segerike, ke go re letšatši le tee ka morago ga Sabatha. Letšatši le le fiwa seka sa tlhompho ka lebaka la gore ke letšatši la tsogo; bona gape le tlaleletšo ya dikanegelo tša tsogo mo dipukung tša Baebangedi (Ditiro 20:7; 1 Bakor. 16:2); gape le 'Letšatši la

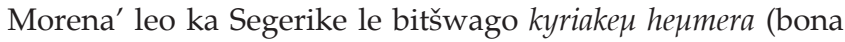
Kut. 1:10).

Ka kakaretšo, tšhupamabaka ya ngwaga wa Bajuta dinakong tša Testamente ye Mpsha (bonnyane morago pele ga 70 AD), e latela kakanyo ya Basatusei, ka gobane ka kakanyo yeo go dirilwe gore ditirelo tša Tempele di sepetšwe ka tshwanelo ka tsela ya molao. Ka tsela yeo letšatši la Tšhologo ya Moyamokgethwa le ile la bewa bjalo ka letšatši la bohlano morago ga tlhagišo ya mathomo ya monyana wa dithakangwaga wa puno ya ngatana ya garase. Taba yeo e bolela gore letšatši la bohlano go tšwa go Lamorena la mathomo morago ga Paseka (bona Lef. 23:15); ke la monyanya woo wa Paseka, woo ka mehla o swanetšego go lebana le letšatši la Lamorena, bjalo ka ge go le bjalo mo tšhupamabakeng ya Sekriste. Kakanyo ya Bafarasei yona, yeo e bilego setlwaedi morago ga AD 70, e hlatholla kgopolo ya 'sabatha', bjalo ka ge go hlalošwa mo go Lef. 23:15 bjalo ka letšatši la monyanya wa Senkgwa sa go hloka komelo, eupša e sego Sabatha sa beke ka beke. Ka wona mokgwa woo letšatši la Tšhologo ya Moyamokgethwa ka mehla le wela letšatšing la go swana mo kgweding (kakanyo ye bohlokwa go bao mahlo a bona e lego leswao la tiragalo ya molao ye e diregago ngwaga ka ngwaga) eupša e sego letšatšing la go swana la beke.

Se bohlokwahlokwa ke go lemoga diphapano tše nnyane tša tšhupamabaka magareng ga Basatusei le Bafarasei tšeo e bilego karogano magareng ga Basatusei le Bafarasei ka lehlakoreng le, le baganyetši ba go tsebega kudu mo go puku ya Jubilees ba go latela tšhupamabaka ya boganyetši, go sa lebalwe gape le dikgopolo tša bjale tša go thewa godimo ga dingwalo tša Khumran, ka go le lengwe. Ge Jesu le barutiwa ba gagwe ba ile ba latela tšhupamabaka ye ya 'boganyetši' go ka be go ile gwa hlathollwa ka fao ba ka bego ba ile ba swara Paseka ka gona pele Jesu a swarwa. Taba yeo e tiišwa ke ge go lemogwa gore baprista ba bagolo le balatedi ba bona ga se ba ka ba latela tšhupamabaka yeo go fihlela morago ga ditlhokofatšo tša gagwe (Jesu) (Jn. 18:28).

\section{Thulaganyo ya dikgwedi}

Bjale go tlo lekolwa thulaganyo ya dikgwedi tša ngwaga go ya ka Bapedi le merafe ya Bibele. Go tlo thongwa ka thulaganyo ya Bapedi pele. Ngwaga wa bogologo o thongwa ka kgwedi ya Mopitlo go ya ka fao re šetšego re boletše ka gona ka godimo. Ka fao ge, dikgwedi tšeo, go ya ka Sehlakwana (wo mongwe wa mebolelwana ya Sepedi) di latelana ka tsela ye:

Go tlo lemogwa gore taba ya peakanyo ya dikgwedi tša ngwaga ka Sepedi, e hlola mathata a thulaganyo ya dikgwedi tša ngwaga ka gobane mebotwana ye mengwe ya go swana le Sekoni le Seroka e na le thulaganyo ye nngwe ya dingwedi tša ngwaga. Bjalo ka ge Sepedi se na le dimmotwana tše ntši, ga se se se makatšago go ba le maina a dikgwedi tša ngwaga a go fapana go ya ka dimmotwana tšeo. Ka go realo, yona taba yeo ya go fapana ga dimmotwana e bolela gore go tlo ba le mathata a go reelwa ga maina a mangwe a dikgwedi tšeo di ka bago le maina a go feta le tee, go ya ka fao go šetšego go boletšwe ka gona ka godimo, go swana le maina a go fapana a dikgwedi tša ngwaga bjalo ka Dibokwane, Hlakola, Mopitlo, Mosegamanye, Phupu le Ngwatobošego, go ya ka tsela yeo dikgwedi tše di sa bitšwego ka go swana go ya ka dimmotwana tša Sepedi. Ke ka yona tsela ye maina a dikgwedi tšeo di selelago, di bontšhitšwego ka godimo, a bitšwago ka go fapana go ya ka dimmotwana tša go fapafapana. Ke go re mebotwaneng ye mengwe ga go dirišwe, mohlala, Ngwatobošego eupša go dirišwa leina la kgwedi ye

Lenaneo la 1: Tatelano ya dikgwedi

\begin{tabular}{lllll}
\hline Tatelano ya dikgwedi & Leina la kgwedi & Tekano ya sebjalebjale ka Seisimane & Sehla & Ditiragalo \\
\hline 1 & Mopitlo & May & Marega & Koma Lehlabula \\
2 & Phato & June & Marega & Koma \\
3 & Phupu/ Ngwatobošego & July & Marega & Koma \\
4 & Mosegamanye & August & Seruthwane & Dikoma di a aloga Mafelong a go fiša mehlare e thoma go khukhuša \\
5 & Lewedi & September & Seruthwane & Menyanya: go folwa ga mabele Diphoofolo di a goela \\
6 & Diphalane & October & Seruthwane & Dipeu di thoma go hloga Diphoofolo di a tswala/goela \\
7 & Dibatsela & November & Selemo & Bophelo bjo bofsa Meletlo ya setšhaba \\
8 & Nthole/Manthole & December & Selemo & Dipula tša matlorotloro \\
9 & Pherekgong & January & Selemo & Go a hlagolwa/meota e a kgatlampana \\
10 & Dibokwane & February & Lehlabula & Menyanya \\
11 & Hlakola & March & Lehlabula & Puno \\
\hline
\end{tabular}


nngwe bjalo ka Phupu, go bjalo le go maineng a dikgwedi tše dingwe tša ngwaga (Hlakola, Mopitlo le Mosegamanye).

Bothata bjoo bja go reela maina a dikgwedi ka ditsela tša go fapana bo tšwelela gape le go merafe ye mengwe ya Bibele bjalo ka Baheberu. Bjale go yo lekolwa ka fao bona ba rulagantšego maina a kgwedi tša ngwaga wa bona ka gona go ya ka tšhupamabaka (ye e fetotšwego gannyane = monyakišiši) ya Seheberu (NBD letl. 158):

\section{Dihla tša ngwaga wa Sepedi}

Ka Sepedi dihla tša ngwaga di lebane le tšhupamabaka ya merero ya bophelo bjalo ka go bjala, go buna, go ntšha dikoma (menyanya le go fola mabele). Ka kakaretšo go ka thwe dihla tša ngwaga wa Sepedi di amanywa le temo ka kakaretšo.

Mopedi o aroganya dihla tše nne tša ngwaga ka go latelana, e lego marega, seruthwane, selemo le lehlabula. Tatelano yeo e bohlokwa ka gobane sehla sa marega se lebane le mathomo a ngwaga wa Sepedi. Go thongwa ka sehla sa marega ka gobane ke nako ya leeto la go thongwa ga go hwa (ga diphedi tša tlhago) le bophelo. Ke go re ke ka nako ya sehla sa marega yeo bophelo bja Mopedi bo fenywago ke lehu.

Le ge maina a dihla tšeo go bonala o ka re a tswalane le maina a dihla tša merafe ye mengwe go swana le Maisimane (marega a lebane le Mopitlo, Moranang le Mosegamanye; seruthwane se lebane le Ngwatobošego, Phupu le Phato, selemo se hlalošwa ke dikgwedi tša Lewedi, Dibatsela le Nthole/ Manthole, mola ka lehlakoreng le lengwe ka lehlabula go bolelwa ka dikgwedi tša Dibokwane, Pherekgong le Hlakola), dipaka tšeo di fapana kudu go ya ka tlhalošo ya setšo sa tšona. Nakong ya marega go ntšhwa dikoma go ya ka fao re šetšego re boletše ka gona ka godimo; seruthwane sona se lebane le paka ya go buna le go fola mabele; ka nako ya selemo gona go a lengwa ka ge dipula di ena, mola lehlabula go ipshinwa ka dithakangwaga le lehlabula ka kakaretšo - ke sehla sa menyanya. Thulaganyo ye ya dihla e thuša Mopedi go beakanya mediro ya diatla le ye mengwe mo bophelong.

\section{Dihla tša ngwaga wa Seheberu}

Ka Seheberu dihla tša ngwaga di amanywa le tšhupamabaka ya temo. Le ge e le gore Baheberu ba amogetše tšhupamabaka ya go thewa godimo ga go reelwa dikgwedi go ya ka lenaneopakatšatši (lunar system), gape bjalo ka balemi, ba bontšha nako ya ngwaga go ya ka sehla, eupša e sego go ya ka maina goba dipalo tša dikgwedi. Ka yona tsela yeo, ngwaga woo kua Palestina o ka aroganywago ka sehla sa komelelo, e lego sa dikgwedi tša Moranang - Lewedi, le sehla sa monola/ dipula, e lego sa dikgwedi tša Diphalane - Hlakola, o ka aroganywa gape ka dikarolwana tša 'go ntšha peu', e lego dikgwedi tša Dibatsela - Manthole le tša 'go buna', e lego Moranang - Phupu (Gn. 8:22).

Ditšhupetšo di bontšha gore baagi ba tikologo goba selete ke bona bathei ba maina a dikgwedi. Mohlala, puno ya korong (Gn. 30:14; Baahl. 15:1) goba garase (barley) (2 Sa. 21:9; Ru. 1:22) e bontšha kgwedi ya Hlakola - Moranang, nakong ya nyooka (earing time) (Eks. 34:21) e ka ba kgwedi ya Hlakola; mola 'dithakangwaga tša morara' (Nu. 13:20) e le kgwedi ya Tammuz ye e amago Phupu - Mosegamanye. Tsheola (pula ya mathomo) (ya go thewa godimo ga tšhupamabaka ya selegae/semmušo ya go thoma ka kgwedi ya Tishri) e na ka dikgwedi tša Lewedi - Diphalane, gomme dipula tša mafelelo di na ka dikgwedi tša Hlakola - Moranang. Seenywa sa selemo, e lego qauyissa dikgwedi tša Phato - Lewedi - leina leo le tšwa go lentšu la 'selemo', gomme gape (leina leo) le

Lenaneo la 2: Tšhupamabaka

\begin{tabular}{|c|c|c|c|c|c|}
\hline$\underline{\text { Kgwedi }}$ & Leina la pele ga bothopša & Leina la ka morago ga bothopša & Tekano ya sebjalebjale & Sehla & Menyanya \\
\hline 1 & $\begin{array}{l}\text { ABIB } \\
\text { Eks. 13:4; 23:15; 34:18; } \\
\text { Dt. 16:1 }\end{array}$ & $\begin{array}{l}\text { NISAN } \\
\text { Est. } 3: 7 \\
\text { Ne. } 2: 1\end{array}$ & $\begin{array}{l}\text { Hlak. - Mor. } \\
\text { (Mar. - Apr.) }\end{array}$ & $\begin{array}{l}\text { Seruthwane } \\
\text { Dipula di a na } \\
\text { Puno ya garase } \\
\text { Puno ya lodi/ folakese }\end{array}$ & $\begin{array}{l}14 \text { Paseke } \\
15-21 \text { Senkgwa sa go hloka } \\
\text { komelo } \\
16 \text { Firastfrit }\end{array}$ \\
\hline 2 & $\begin{array}{l}\text { ZIV } \\
1 \text { Dikg. 6:1, } 37\end{array}$ & IYYAR & $\begin{array}{l}\text { Mor. - Mop. } \\
\text { (Apr. - May) }\end{array}$ & Mathomo a sehla sa komelelo & 14 Paseka ya ka morago \\
\hline 3 & - & $\begin{array}{l}\text { SIYAN } \\
\text { Est. 8:9 }\end{array}$ & $\begin{array}{l}\text { Mop. - Phup. } \\
\text { (May-June) }\end{array}$ & Mago a mathomo a a butšwa & $\begin{array}{l}6 \text { Tšhollo ya Moyamokgethwa } \\
\text { Monyanya wa Dibeke } \\
\text { Puno }\end{array}$ \\
\hline 4 & - & TAMMUZ & $\begin{array}{l}\text { Phup. - Mos. } \\
\text { (June - July) }\end{array}$ & Puno ya diterebe & - \\
\hline 5 & - & $A B$ & $\begin{array}{l}\text { Mos. - Phat. } \\
\text { (July - Aug.) }\end{array}$ & Puno ya dihlware & - \\
\hline 6 & - & $\begin{array}{l}\text { ELUL } \\
\text { Ne. 6:15 }\end{array}$ & $\begin{array}{l}\text { Phat. - Lew. } \\
\text { (Aug. - Sept.) }\end{array}$ & Ditatele le mago a selemo & - \\
\hline 7 & $\begin{array}{l}\text { ETHANIM } \\
1 \text { Dikg. 8:2 }\end{array}$ & TISHRI & $\begin{array}{l}\text { Lew. - Diph. } \\
\text { (Sept. - Oct.) }\end{array}$ & Dipula tša mathomo & $\begin{array}{l}1 \text { Diprompeta } \\
10 \text { Letšatši la Kgwerano } \\
15-21 \text { Ditente } \\
22 \text { Kgobokano ya kgonthe }\end{array}$ \\
\hline 8 & $\begin{array}{l}\text { BUL } \\
1 \text { Dikg. 6:38 }\end{array}$ & MARCHESYAN & $\begin{array}{l}\text { Diph. - Dib. } \\
\text { (Oct. - Nov.) }\end{array}$ & Temo ya marega ya mago & - \\
\hline 9 & - & $\begin{array}{l}\text { CHISLEY } \\
\text { Ne. } 1: 1\end{array}$ & $\begin{array}{l}\text { Dib. - Man. } \\
\text { (Nov. - Dec.) }\end{array}$ & Go bjala & 25 Boikgafo \\
\hline 10 & - & $\begin{array}{l}\text { TEBETH } \\
\text { Est. 2:16 }\end{array}$ & $\begin{array}{l}\text { Man. - Pher. } \\
\text { (Dec. - Jan.) }\end{array}$ & Dipula (lehlwa dinagagodimo) & - \\
\hline 11 & - & $\begin{array}{l}\text { SHEBAT } \\
\text { Zac. 1:7 }\end{array}$ & $\begin{array}{l}\text { Pher. - Dibo. } \\
\text { (Jan. - Feb.) }\end{array}$ & Matšoba a amantele & - \\
\hline 12 & - & $\begin{array}{l}\text { ADAR } \\
\text { Est. 3:7 }\end{array}$ & $\begin{array}{l}\text { Dibo. - Hlak. } \\
\text { (Feb. - Mar.) }\end{array}$ & Puno ya dinamune & - \\
\hline
\end{tabular}


tšwa go lentšu la 'phišo'. Digwedi tša T\ebet le Sðebat \di bonwa bjalo ka dikgwedi tša go tonya (marega).

Go ya ka methopo ya Testamente ye Tala, thulaganyo ye bjalo ya mabaka e ka bapetšwa le tšhupamabaka ya temo ya go thalwa leswikeng, ya go humanwa kua Getser ka ngwaga wa 1908, yeo go dumelwago gore sethalwa seo se thadilwe ke mošemanyana wa sekolo mo ngwagakgolong wa bolesome Pele ga Kriste. Phetolelo ya gona ga e tsebege, eupša e tšweletša ditshepedišo tša temo tša dikgwedi tša ngwaga tše lesomepedi tša go thoma ka sehla sa lehlabula:

Two months of storage. Two months of sowing. Two months of spring growth. Month of pulling flax. Month of barley harvest. Month when everything (else) is harvested. Two months of pruning (vines). Month of summer fruit .

(cf. DOTT 201-203)

Tšhupamabaka yeo e gatelela bohlokwa bja dikarolo tša ngwaga tše seswai, e lego dikgwedi tše pedi tša go boloka ka dišegong, tše pedi tša puno ya garase, tše pedi tša go bjala, e tee ya go fetša go buna, tše pedi tša go thenela mehlare le e tee ya seenywa sa selemo.

Go ka rungwa ka go re ditsela tše dingwe tša go bontšha mabaka le dihla di akaretšwa ke mantšu a go nepiša 'paka'

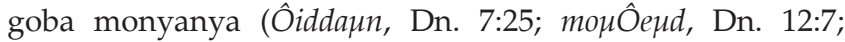
$z^{e}$ maun, Mmol. 3:1; Ne. 2:6), bona gape le Ps. 104:27. Gantši ditiragalo tša histori le tšona di rulaganywa go ya ka mengwaga ya pušo ya babuši goba ka tsela ya go nyalanya le tiragalo ya go tuma ya setšhaba le mabaka, bjalo ka leeto la Baisraele la go tloga Egipeta go leba Kanana, go dula ga Baisraele kua Egipeta (Eks. 12:40); go agwa ga Ntlokgethwa ya mathomo (1 Dikg. 6:1); goba mengwaga ye masomešupa ya Bothopša kua Babeloni (Esk. 33:21); goba tšhišinyego ya lefase nakong ya pušo ya Usia (Am. 1:1; Sk. 14:5).

\section{Tlhalošo ya maina a dikgwedi tša ngwaga wa Sepedi}

Tharollo ya bothata bjo e tlo nepišwa ka go lekola modu, setlogo goba tlholego ya maina a dikgwedi tša ngwaga mo polelong ya Sepedi. Nyakišišo e lemogile gore (1) go na le mebotwana ye mengwe yeo e reetšego maina a dikgwedi tša ngwaga ka ntle ga tlhalošo ye e rilego, goba mohlomongwe tlhalošo ye bjalo ga e sa tsebja mebotwaneng yeo, le (2) mola ka lehlakoreng le lengwe go na le mebotwana ye mengwe yeo yona e nago le thalošo ye e tiilego ya go reelwa ga maina a dikgwedi tša ngwaga.

Godimo ga moo maina a a dikgwedi a a retwa ka gobane a bohlokwa mo setšong le bophelong bja Mopedi. Mopedi o tšweletša se sengwe le se sengwe ka theto, gagolo ge e le se bohlokwahlokwa bophelong bja gagwe. Bjale go yo hlokomelwa tlhalošo yeo ya maina a dikgwedi tša ngwaga go ya ka Mokgalabje Lethole Tau le Mokgekolo Mologadi NgwanaMagolego.

\section{Mopitlo}

Mopitlo ke kgwedi ya mathomo ya ngwaga wa Bapedi. Go thwe, Mopitlo nka putlaganya selemo le marega. Go era gore kgwedi ye e kgaogantšha selemo le marega. Ke kgwedi ya mathomo ya sehla sa marega. Phefo e a hlaba kgweding ye gomme dibjalo le dimela di thoma go sehlefala ka lebaka la go tonya. Mopitlo ke kgwedi ya go loma ngwaga. Ke nakong ye batho ba itiago mpa ka lenono. Mahea a a bunwa. Merogo ya thoma mošomo wa go fola gomme mabele a lotwa ka dišegong. Ye ke kgwedi ya lethabo, menyana le meketeko.

Mopitlo ke kgwedi ye ka yona Naka e hlabago. Batho ba tsoga bošego go emela Naka ge e hlaba ka gobane go na le mmolelo le tumelo ya go re motho wa mathomo wa go bona Naka e hlaba o ba le mašoto. Ge Naka e seno hlaba, motho wa go e bona pele o begela kgoši ka ge Naka e le sešupo sa mmakgonthe sa go eletša kgoši go ntšha koma, le gore kgoši e thome sehla se sefsa sa ngwaga ka mafolofolo. Ge e retwa go thwe:

Mopitlo nka putlaganya selemo le marega,

Ke Mopitlo mopitla a putla,

O putla marega le lehlabula.

\section{Phato}

Ye ke kgwedi ya bobedi ya ngwaga wa Sepedi. Mehlare e uša/fofora matlakala, gagolo Mogaba o bonagala o le wo moserolwana. Le ge go le bjalo matlakala a mehlare ye mengwe a sa tagile, ga ešita le matšoba a yona e sa le a mabotse gagologolo dinageng tša go fiša. Mabjang a mangwe le ona a thoma go fetola mmala, gagolo mafelong a go tonya. Dienywa di thoma go huba. Dinong di tletše sebakeng. Go thomile go sesefala. Dipeu di thoma go oma. Naga e thoma go welwa ke kgwakgwa. Dilo ka moka tša tlhago di thoma go sesefalelwa ke bophelo. Ge kgwedi ye e retwa go thwe:

Ke Phato phatola dihlare,

Ke Mangwetši 'a Mogale,

Ke Phato kgwedi 'a selemomarega.

\section{Phupu/Ngwatobošego}

Ka gare ga mmotwana o tee kgwedi ye e na le maina a mabedi, e lego Phupu le Ngwatobošego. Godimo ga moo thalošo ya kgwedi ye e na le go thulana le ya Lewedi. Le ge go le bjalo nyakišišo e yo kopanya ditlhalošo tšeo mmogo. Ke kgwedi ya go folwa ga mabele a bunwa mathomong. Kgwedi ye e amanywa le nonyana ya go bitšwa mankgodi. Le ge e fofa godimo sebakeng e kgona go bona le tša kua fase. Ke nakong ye mohuta wa dinonyana tše o bonalago ka boati sebakeng go swara leeto. Ge e eya swiswing manong le ona a thoma go fokotšega sebakeng. Ke kgwedi ye maakabosana a khudugelago nagadikgole / mafelong a mangwe a go fiša ka lebaka la boemo bja meso (marega). Ke ka fao ge e retwa go thwego:

Mankgodi a manong a ile phupu,

Phupu e fuputša dipeu tša selemo,

Manong a boa ka lela ka lenaka la kgoši,

Go thwe a be a ile phupu.

Ke manong maremane,

Maremane wa pelo tša balemi. 


\section{Mosegamanye}

Ke kgwedi ye mehlare ya mathomo e thomago go khukhuša, gagolo mengana. Diphoofolo di thoma go kgotha matlakala a ditala tšeo di thomago go hloga. Mo le mola go thoma go ruthela ka gobane marega a thoma go fela. Go na le bošaetšana bjo bonnyane mo tlhalošong ka gore bogolo bja naga ga go na botalana lebakeng le. Gantši, le ge mengwaga e sa swane ke nako ya komelelo / kgwakgwa. Mehlare e ka se khukhuše ka nako ye ka ge go sa tonya kudu. Mehlare e khukhuša ge go thoma go ruthela. Le ge go le bjalo nyakišišo e amogela tlhalošo ye ka gobane e hlatholla ditiragalo tše di rilego tša kgwedi ye, gagolo ka gore mafelong a go fiša go na le kgonagalo ya gore go ka ba mehlare ye e ka khukhušago. Ge kgwedi ye e retwa go thwe:

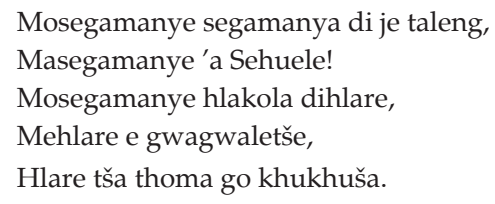

\section{Lewedi}

Ye ke kgwedi ya seruthwane. Batho ba kgothatšwa ke go goroga ga sehla se sefsa sa ngwaga sa go kgabiša naga. Diphedi ka moka tša go goela marega di tšwelela gape. Digagabi, gagolo dinoga, di thoma go sabalala le naga. Batho ba itokišetša bophelo bjo bofsa. Ge e retwa go thwe:

Ke Lewedi la dikhukhwanyane,

Ngwetabošego ke a foafoa;

Ke Lewedi 'a dibatsela a di phalale.

\section{Diphalane}

Ye ke kgwedi ya dinonyana. Naga e thoma go tshotshoma ka melodi ya dinonyana. Naga e aparelwa ke botalana le ka mebala ya go taga ya mehutahuta ya matšoba. Ye ke nako ya go swarwa meletlo ya setšhaba ya go fapafapana ya go amogela sehla se sefsa. Ke kgwedi ya mathomo ya selemo ya go lema. Go thoma go letelwa dipula tša mathomo. Ke kgwedi ya lethabo go Mopedi ebile ke nako ya lethabo go diphoofolo, gagolo diphala. Go bonala nako ye diphala di tswala di bile di kgaramiša diphalana. Ge e šetše e retwa go thwe:

Ke Diphalane tša madikaphalana,

Ke Diphalane tša phalana,

Ke Diphalane tša matšema,

Matšema a rerwa sentsokela.

\section{Dibatsela}

Ye ke kgwedi ya go tsebja ka dipula tša matlorotloro. Ke nako ya go falala ga megobe. Ditsela di runya meetsemagakwa. Ke mehlaka le megobe mo gofela. Dipula di thomile go falala. Meetse a tšama a ema didibana e le meetsemagakwa mo gohle le mo ditseleng. Naga e tshotshoma methopo le mehlaka, mo gohle go lla digwagwa le dikuukuu molapong. Go kwagala meledilodi ya dinonyana dikgweng; ebile ke nako ye botse ya go swara / tanya ga batsomi. Bontši bja mehlare bo goromana ka dienywa, gomme batho ba fula ka go rata. Ge ba e reta ba re:
Dibatsela kgwedimmamoratwa,

Dibatsela di phalala lephalalo,

Legohle phamphangmeetsemagakwa,

Mo go tlago Dibatsela' tholwana.

\section{Manthole/Nthole}

Kgwedi ye e amana le go rolwa merwalo. Nako ye ya ngwaga go rolwa lehlabula, le ge e le gore go na le mebotwana ye mengwe e bolelago gore go rolwa dipeu ka gore go thwe 'nthole dipeu di leswe'. Taba yeo e bolela gore ka nako ye go lema go phethilwe. Mo gape go lemogwa thulano ya mebotwana malebana le go reelwa ga leina la kgwedi ye ya ngwaga. Bjalo ka ge mebotwana ye mentši e gatelela lehlabula, kgwedi ye e yo lebanywa le ya lehlabula, ka gobane go thwe:

Mme nthole maraka,

Kgwedi ke ile ke fetile'.

Mmolelwana (Temanatheto) wo o gatelela gore nakong ye go lewa lehlabula. Ka tsela yeo nthole ye e nepiša go rolwa dintsho, mahea, dinawa, maraka le tše dingwe tša ka mašemong. Basadi ba rola barwedi ba bona merwalo ya lehlabula.

\section{Pherekgong}

Leina la kgwedi ye le amanywa le dilo tše pedi, e lego (1) leeba la go bitšwa mokurwane le (2) puno ya dinawa. Kgwedi ye e nyalelane le mohuta wo wa maeba ka gobane nakong ye ya ngwaga maeba ao a swaragane le go topa le go aga dihlaga tša ona ka diphatana tša go oma tša mehlašana. Ka go realo Pherekgong e amanywa le madulo (sehlaga). Ke kgwedi ye borutho ka gobane e tswalane le legae (sehlaga) la kgodišo ya mafotwana a maeba.

Godimo ga moo, Pherekgong e sepelelana le puno ya dinawa. Nako ya kgwedi ye ke ya go bunwa ga dinawa. Ka fao e tsebega ka mokhora ka gobane ke nakong yeo dijo (sa go šeba e lego setopša le semotwane) di sa hlokwego. Ka lehlakoreng le lengwe le maeba a iphepa ka dinawa tšeo. Dinawa ke se sengwe sa dijo tše bohlokwa maphelong a Bapedi. Ka fao Pherekgong le dinawa ke kgeswa le šago. Ke ka fao Mopedi a nyalanyago Pherekgong le mokhora; a be a fetše ka go e reta ka go re:

Pherekgong 'a dinawa

Ke balame Ngoato 'a Ngoatwana,

Ke šuthela dibokwane tša morula.

\section{Dibokwane}

Kgweding ye ke nakong ya ge dibokwana (go tšwa go diboko) di bonalago ka bontši gagolo mo dienyweng. Ke nako ya go butšwa/raša ga marula le dikenywa tše dingwe tša naga le tša ka mašemong. Batho ba ipshina ka bjala bja morula ka gore e ba bjo bontši nakong ye. Ge e šetše e reteletšwa bohlokwa bja yona maphelong a Bapedi go thwe:

Ke dibokwana tša marula,

Se mphe ke khoše. 


\section{Hlakola}

Lentšu le 'go hlakola' le lebane le go thopa/amoga. Hlakola ke kgwedi ya meila. Ga go dirwe menyanya mo motseng. Kgwedi ye e amana le go longwa ga ngwaga ka gobane ke mathomo a go ja lehlabula - mo le mola ba buna ditema tša pele, mola ba bangwe ebile ba fola mabejana a go thoma go butšwa. Ke kgwedi ya go hlaba ga Senakane. Bophelong bja Mopedi ke nako ya kotsi. Naga e thoma go sehlefala ka lebaka la go tonya, gagolo ga mesong le mantšiboa/bošego.

Hlakola ke kgwedi ya go tuma ka diila le dibeela. Ga go meletlo yeo e swarwago go swana le koma, manyalo le dikgoro tša kahlolo. Ge madira a ka romelwa ntweng nakong ye, go letetšwe gore a ka se fenye, eupša a tlo fenywa ke manaba a ona.

Ge kgwedi ye e retwa go thwe:

Ke Hlakola' Matsepe,

Ba re mogolo o raga ka sefularo,

Hlakola ke mogale,

Mogale o raga ka sefularo.

Ke Hlakola mohlakolane wa matšepe,

Mogale o raga ka sefularo,

Hlakola hlakola dihlare,

Ke hlakotše mogatša' kgoši ditšwalo.

\section{Moranang}

Moranang ke kgwedi ya mafelelo ya ngwaga wa Sepedi. E amanywa le go thongwa ga go tonya ga phefšana ya marega. E sa le kgwedi ya go ntšhwa ga dikoma (go kgotha). Gape e amanywa le go thoma go folwa ga mabele. Ye ke kgwedi ye le yona go thwego e aroganya lehlabula le marega. Ke ka lebaka le e retwago ka go re:

Moranang wa kgomo (pelo) tša badiši,

$\mathrm{O}$ a fiša, o borutho;

Kgwedi a o maruru,

A o marutho?

Go a fiša go a tonya. Nakong ye go dijo di se kae fela tše di ka kgewago bjalo ka ge e le lehlabula, goba tša bunwa le go lotwa ka dišegong.

\section{Thumo}

Le ge go na le phapano ye e rilego ya thalošo ya dikgwedi tša ngwaga wa Sepedi le Seheberu eupša go lemogwa kwano ye kgolo magareng ga dipolelo tše pedi tšeo. Kwano ya mathomo ke ya palo ya dikgwedi le gore mengwaga ya bona e thongwa ka sehla sa go swana, e lego sa lehlabula. Godimo ga moo maina a dikgwedi tša ngwaga tša Seheberu tše nne (4), e lego Abib, Ziv, Ethanim le Bul, ao a bego a dirišwa pakeng ya pele ga bothopša, ao a ilego a se hlwe a sa dirišwa pakeng ya ka morago ga bothopša, a fapana le ao a dirišwago morago ga bothopša. Ka lehlakoreng le lengwe ponagalo ye bjalo e tšwelela gape mo polelong ya Sepedi fao maina a dikgwedi, ao a nago le leina la go feta le tee bjalo ka Dibokwane, Hlakola, Mopitlo, Mosegamanye, Phupu le Ngwatobošego go ya ka thulaganyo ya mebotwana ya Sepedi a dirišwago mo tšhupamabakeng. Ka fao thulaganyo ye bjalo e tswala kwano ye e rilego ya tšhupamabaka ya magareng ga dipolelo tše.

Le ge go le bjalo tharollo ya taba yeo ya maina a go fapana a dikgwedi tša Sepedi e tla thewa godimo ga motheo wa tekanyetšo (standardisation) ya maina a dikgwedi tša ngwaga go ya ka mebotwana ya go fapafapana. Tekanyetšo ye bjalo e tlo dirwa go ya ka bokgafetšakgafetšo (frequency) bja tirišo ya maina a dikgwedi magareng ga mebolelwana ya go fapana ya Sepedi.

\section{Methopo}

Charles, R.H., 1913, The Book of Jubilees from the Apocrypha and Pseudepigrapha of the Old Testament, Clarendon Press, Oxford, UK.

Bickerman, E.J., 1968, Chronology of the ancient world, Cornell University Press, New York.

Dancy, J.C., 1954, Commentary on I Maccabees, Blackwell, Oxford, UK.

Geldenhuys, N., 1950, Commentary on the Gospel of Luke: the English text with introduction, exposition and notes, Eerdmans, Grand Rapids, MI.

Finegan, J., 1964, Handbook of Bible chronology: principles of time reckoning in the ancient world and problems of chronology in the Bible. N.J. Princeton University Press, Princenton.

Jaubert, A., 1957, 'La Date de la Cène, and “Jesu et le calendrier de Qumrân"' NTS 7 , 1960-1961, pp. $1 \mathrm{ff}$.

Milik, J.T. (ed.), 1976, The Books of Enoch: Aramaic Fragments of Qumran Cave 4, Library of Congress, Oxford, UK.

Mönnig, H.O., 1988, The Pedi, J.L. van Schaik, Pretoria.

O'Neil, W.M., 1975, Time and the Calendars, Sydney University Press, Sydney, N.S.W.

Segal, J.B., 1963, The Hebrew Passover from the earliest times to AD, Oxford University Press, New York.

Van Goudoever, J., 1959, Biblical Calendars, Brill, Leiden.

Wiesenberg, E.J., 1971, 'Calender', in C. Roth (ed.), Encyclopadia Judaica, n.p., MacMillan, New York.

Wiesenberg, E.J., 2008, Bibele (ya Mangwalo a Makgethwa a Testamente ye Tala le a Testamente ye Mpsha), Bible Society of South Africa, Bellville.

Wiesenberg, E.J., 1962, The New Bible Dictionary, Tyndale House Publishers, Inc., Wheaton, IL. 